\title{
A SIMPLE PROOF OF WATSON'S PARTITION CONGRUENCES FOR POWERS OF 7
}

\author{
F. G. GARVAN \\ (Received 30 August 1982, revised 26 November 1982) \\ Communicated by J. H. Loxton
}

\begin{abstract}
Ramanujan conjectured that if $n$ is of a specific form then $p(n)$, the number of unrestricted partitions of $n$, is divisible by a high power of 7. A modified version of Ramanujan's conjecture was proved by G. N. Watson.

In this paper we establish appropriate generating formulae, from which Watson's results follow easily.

Our proofs are more straightforward than those of Watson. They are elementary, depending only on classical identities of Euler and Jacobi. Watson's proofs rely on the modular equation of seventh order. We also need the modular equation but we derive it using the elementary techniques of $O$. Kolberg.
\end{abstract}

1980 Mathematics subject classification (Amer. Math. Soc.): 10 A 45; Secondary 10 D 23.

\section{Introduction}

In 1919 Ramanujan [6] conjectured that if $\alpha \geqslant 1$, if $\delta_{\alpha}$ is the reciprocal modulo $5^{\alpha}$ of 24 and if $\lambda_{\alpha}$ is the reciprocal modulo $7^{\alpha}$ of 24 , then

$$
p\left(5^{\alpha} n+\delta_{\alpha}\right) \equiv 0 \bmod 5^{\alpha}
$$

and

$$
p\left(7^{\alpha} n+\lambda_{\alpha}\right) \equiv 0 \bmod 7^{\alpha}
$$

This article comprises, in the main, the first chapter in the author's Masters thesis (University of N.S.W., 1982).

(C) 1984 Australian Mathematical Society $0263-6115 / 84 \$ A 2.00+0.00$ 
In each case he proved his conjecture for $\alpha=1$ and 2. In 1938 Watson [7] proved (1.1) for general $\alpha$. Watson's proof has been simplified by many writers of which the most recent are Hirschhorn and Hunt [4].

Chowla [2] noticed that (1.2) fails for $\alpha=3$. In fact from Gupta [3] we have

$$
p\left(\lambda_{3}\right)=p(243)=133978259344888,
$$

which is divisible by $7^{2}$ but not by $7^{3}$. Watson [7] proved the appropriate modification of (1.2), namely that if $\beta \geqslant 1$ then

$$
p\left(7^{2 \beta-1} n+\lambda_{2 \beta-1}\right) \equiv 0 \bmod 7^{\beta}
$$

and

$$
p\left(7^{2 \beta} n+\lambda_{2 \beta}\right) \equiv 0 \bmod 7^{\beta+1} .
$$

Watson also proved that if $\beta \geqslant 1$, then

$$
\begin{aligned}
p\left(7^{2 \beta} n+\lambda_{2 \beta}-4.7^{2 \beta-1}\right) & \equiv p\left(7^{2 \beta} n+\lambda_{2 \beta}-2.7^{2 \beta-1}\right) \\
& \equiv p\left(7^{2 \beta} n+\lambda_{2 \beta}-7^{2 \beta-1}\right) \equiv 0 \bmod 7^{\beta+1} .
\end{aligned}
$$

Watson proved (1.3) and (1.4) using the modular equation of seventh order. We also need the modular equation but we derive it using the elementary techniques of O. Kolberg [5]. The remainder of our proof of (1.3) is analogous to that of Hirschhorn and Hunt. Our main result stated below contains an algorithm for calculating the coefficients in the generating functions for $p\left(7^{\alpha} n+\lambda_{\alpha}\right)$. We carry out these calculations for $\alpha=1,2$.

It is worth noting that Atkin [1] has generalised (1.1) and (1.3) to the functions $p_{-k}(m)$. Atkin has proved his congruences in detail for powers of 5 but has only sketched briefly the basic formulae required for certain power primes. Here $p_{-k}$ is defined by

$$
\sum_{n \geqslant 0} p_{-k}(n) q^{n}=\prod_{n \geqslant 1}\left(1-q^{n}\right)^{-k}
$$

so that $p_{-1}(n)=p(n)$.

Our main result is

THEOREM (1.6). If $\alpha \geqslant 1$, then

$$
\sum_{n \geqslant 0} p\left(7^{\alpha} n+\lambda_{\alpha}\right) q^{n}= \begin{cases}\sum_{i \geqslant 1} x_{\alpha, i} q^{i-1} \frac{E\left(q^{7}\right)^{4 i-1}}{E(q)^{4 i}}, & \alpha \text { odd }, \\ \sum_{i \geqslant 1} x_{\alpha, i} q^{i-1} \frac{E\left(q^{7}\right)^{4 i}}{E(q)^{4 i+1}}, & \text { a even, }\end{cases}
$$




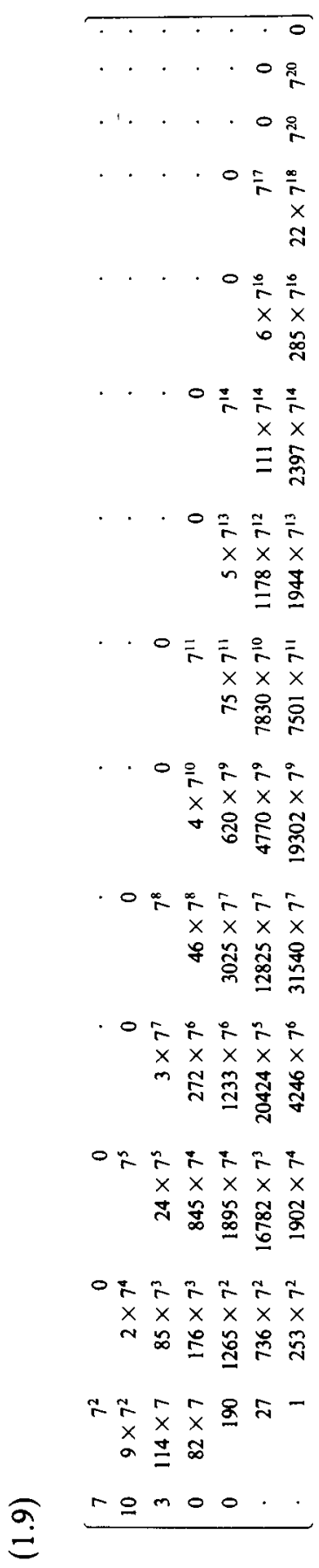


where $E(q)=\Pi_{n>1}\left(1-q^{n}\right), \mathbf{x}_{1}=(7,49,0,0, \ldots)$, and for $\alpha \geqslant 1$,

$$
\mathbf{x}_{\alpha+1}= \begin{cases}\mathbf{x}_{\alpha} A, & \alpha \text { odd }, \\ \mathbf{x}_{\alpha} B, & \alpha \text { even } .\end{cases}
$$

Here $A=\left(a_{i, j}\right)_{i, j>1} B=\left(b_{i, j}\right)_{i, j \geqslant 1}$ are defined by

$$
a_{i, j}=m_{4 i, i+j}, \quad b_{i, j}=m_{4 i+1, i+j},
$$

The first seven rows of $M$ are given in (1.9), and for $i \geqslant 4 m_{i, 1}=0$, for $i \geqslant 8$ $m_{i, 2}=0$ and for $i \geqslant 8, j \geqslant 3$,

$$
\begin{aligned}
m_{i, j}= & 7 m_{i-3, j-1}+35 m_{i-2, j-1}+49 m_{i-1, j-1} \\
& +m_{i-7, j-2}+7 m_{i-6, j-2}+21 m_{i-5, j-2}+49 m_{i-4, j-2} \\
& +147 m_{i-3, j-2}+343 m_{i-2, j-2}+343 m_{i-1, j-2} .
\end{aligned}
$$

We need some preliminary results.

Lemma (2.1).

$$
E(q)=E\left(q^{49}\right)\left[Q_{0}-q Q_{1}-q^{2}+q^{5} Q_{5}\right],
$$

where $Q_{0}, Q_{1}$ and $Q_{5}$ are power series in $q^{7}$ which satisfy

$$
\left\{\begin{array}{c}
Q_{0} Q_{1}^{2}-Q_{0}^{2}+q^{7} Q_{5}=0 \\
Q_{0}-Q_{1}^{2}-q^{7} Q_{1} Q_{5}^{2}=0 \\
Q_{0}^{2} Q_{5}-q^{7} Q_{5}^{2}-Q_{1}=0 \\
Q_{0} Q_{1} Q_{5}=1
\end{array}\right.
$$

Proof. Here we assume

$$
E(q)=\sum_{-\infty}^{\infty}(-1)^{n} q^{\left(3 n^{2}-n\right) / 2}
$$

and

$$
E(q)^{3}=\sum_{n \geq 0}(-1)^{n}(2 n+1) q^{\left(n^{2}+n\right) / 2} \quad(\mathrm{Jacobi}) .
$$

Write $E(q)=E_{0}+E_{1}+E_{2}+E_{3}+E_{4}+E_{5}+E_{6}$ where $E_{i}$ contain those terms of $E(q)$ in which the power of $q$ is congruent to $i \bmod 7$. 
Since $\frac{1}{2}\left(3 r^{2}-r\right) \not=3,4$ and $6 \bmod 7$,

$$
E_{3}=E_{4}=E_{6}=0 \text { and } E(q)=E_{0}+E_{1}+E_{2}+E_{5} .
$$

Now

$$
\begin{aligned}
E_{2}= & \sum_{\substack{\left(3 r^{2}-r\right) / 2 \equiv 2 \\
\bmod 7}}(-1)^{r} q^{\left(3 r^{2}-r\right) / 2}=\sum_{\substack{r \equiv 6 \\
\text { mod } 7}}(-1)^{r} q^{\left(3 r^{2}-r\right) / 2} \\
= & \sum_{-\infty}^{\infty}(-1)^{7 n-1} q^{\left[147 n^{2}-49 n+4\right] / 2}=-q^{2} \sum_{-\infty}^{\infty}(-1)^{n}\left(q^{49}\right)^{\left(3 n^{2}-n\right) / 2} \\
= & -q^{2} E\left(q^{49}\right) . \\
E(q)^{3}= & \left(E_{0}+E_{1}+E_{2}+E_{5}\right)^{3} \\
= & \left(E_{0}^{3}+6 E_{0} E_{2} E_{5}+3 E_{1}^{2} E_{5}\right)+\left(E_{5}^{3}+3 E_{0}^{2} E_{1}+6 E_{1} E_{2} E_{5}\right) \\
& +\left(3 E_{0} E_{1}^{2}+3 E_{0}^{2} E_{2}+3 E_{2}^{2} E_{5}\right)+\left(E_{1}^{3}+6 E_{0} E_{1} E_{2}+3 E_{0} E_{5}^{2}\right) \\
& +\left(3 E_{0} E_{2}^{2}+3 E_{1}^{2} E_{2}+3 E_{1} E_{5}^{2}\right) \\
& +\left(3 E_{0}^{2} E_{5}+3 E_{2} E_{5}^{2}+3 E_{1} E_{2}^{2}\right)+\left(E_{2}^{3}+6 E_{0} E_{1} E_{5}\right) \\
= & \sum_{n \geqslant 0}(-1)^{n}(2 n+1) q^{\left(n^{2}+n\right) / 2},
\end{aligned}
$$

and since $\frac{1}{2}\left(n^{2}+n\right) \not 2,4$ and $5 \bmod 7$ we have

$$
\left\{\begin{array}{l}
3 E_{0} E_{1}^{2}+3 E_{0}^{2} E_{2}+3 E_{2}^{2} E_{5}=0, \\
3 E_{0} E_{2}^{2}+3 E_{1}^{2} E_{2}+3 E_{1} E_{5}^{2}=0 \\
3 E_{0}^{2} E_{5}+3 E_{2} E_{5}^{2}+3 E_{1} E_{2}^{2}=0 .
\end{array}\right.
$$

If we define $Q_{0}, Q_{1}$ and $Q_{5}$ by

$$
E_{0}=E\left(q^{49}\right) Q_{0}, \quad E_{1}=-q E\left(q^{49}\right) Q_{1} \quad \text { and } \quad E_{5}=q^{5} E\left(q^{40}\right) Q_{5}
$$

then $Q_{0}, Q_{1}$ and $Q_{5}$ are power series in $q^{7}$. (2.2) follows from (2.4) and we have

$$
E(q)=E\left(q^{49}\right)\left[Q_{0}-q Q_{1}-q^{2}+q^{5} Q_{5}\right] .
$$

Multiplying the first equation in (2.2) by $Q_{5}$ and substituting $Q_{0}^{2} Q_{5}=Q_{1}+q^{7} Q_{5}^{2}$ we obtain

$$
Q_{0} Q_{1}^{2} Q_{5}=Q_{1}
$$

or

$$
Q_{0} Q_{1} Q_{5}=1 \text {, which is }(2.3)
$$


In fact, it can be shown that

$$
\begin{aligned}
& Q_{0}=\prod_{n \geqslant 1} \frac{\left(1-q^{49 n-14}\right)\left(1-q^{49 n-35}\right)}{\left(1-q^{49 n-7}\right)\left(1-q^{49 n-42}\right)}, \\
& Q_{1}=\prod_{n \geqslant 1} \frac{\left(1-q^{49 n-21}\right)\left(1-q^{49 n-28}\right)}{\left(1-q^{49 n-14}\right)\left(1-q^{49 n-35}\right)}, \\
& Q_{5}=\prod_{n \geqslant 1} \frac{\left(1-q^{49 n-7}\right)\left(1-q^{49 n-42}\right)}{\left(1-q^{49 n-21}\right)\left(1-q^{49 n-28}\right)},
\end{aligned}
$$

using Watson's quintuple product identity.

LEMMA (2.5). If $\omega^{7}=1, \omega \neq 1$ then

$$
E(q) E(\omega q) E\left(\omega^{2} q\right) E\left(\omega^{3} q\right) E\left(\omega^{4} q\right) E\left(\omega^{5} q\right) E\left(\omega^{6} q\right)=\frac{E\left(q^{7}\right)^{8}}{E\left(q^{49}\right)}
$$

PROOF.

$$
\begin{aligned}
\prod_{k=0}^{6} E\left(\omega^{6} q\right) & =\prod_{n \geqslant 1}\left(1-q^{n}\right)\left(1-\omega^{n} q^{n}\right)\left(1-\omega^{2 n} q^{n}\right) \cdots\left(1-\omega^{6 n} q^{n}\right) \\
& =\prod_{\substack{n \geqslant 1 \\
n \equiv 0 \bmod 7}}\left(1-q^{n}\right)^{7} \prod_{\substack{n \geq 1 \\
n \neq 0 \bmod 7}}\left(1-q^{7 n}\right) \\
& =\prod_{n \geqslant 1}\left(1-q^{7 n}\right)^{7} \frac{\prod_{n \geqslant 1}\left(1-q^{7 n}\right)}{\prod_{\substack{n \geqslant 1 \\
n \equiv 0 \bmod 7}}\left(1-q^{7 n}\right)} \\
& =\frac{\prod_{n \geqslant 1}\left(1-q^{7 n}\right)^{8}}{\prod_{n \geqslant 1}\left(1-q^{49 n}\right)}=\frac{E\left(q^{7}\right)^{8}}{E\left(q^{49}\right)} .
\end{aligned}
$$

The main result of this section is Lemma (3.1). Our proof relies on the modular equation of seventh order (3.14), which appears in Watson's paper but which we obtain by elementary method.

We now introduce the operators $H_{i}, 0 \leqslant i \leqslant 6$ which act on a series of powers of $q$ and simply pick out those terms in which the power of $q$ is congruent to $i$ modulo 7. Set $H=H_{0}$. 
322

F. G. Garvan

[7]

LEMMa (3.1). For $i \geqslant 1$,

$$
H\left(\xi^{-i}\right)=\sum_{j \geq 1} m_{i, j} T^{-j}
$$

where

$$
\xi(q)=\frac{E(q)}{q^{2} E\left(q^{49}\right)}, \quad T(q)=\frac{E\left(q^{7}\right)^{4}}{q^{7} E\left(q^{49}\right)^{4}}
$$

and the $m_{i, j}$ are defined by (1.9) and (1.10).

We leave the proof of Lemma (3.1) till later. As an immediate consequence we have the following lemma.

LEMMA (3.4). For $i \geqslant 1$,

$$
H\left(\xi^{-4 i}\right)=\sum_{j \geq 1} a_{i, j} T^{-i-j}
$$

and

$$
H\left(\xi^{-(4 i+1)}\right)=\sum_{j \geq 1} b_{i, j} T^{-i-j}
$$

where the $a_{i, j}, b_{i, j}$ are defined by (1.8).

Proof. It is easy to check that $H\left(\xi^{-4 i}\right)$ as a polynomial in $T^{-1}$ has no terms of degree $i$ or less. So by Lemma (3.1)

$$
H\left(\xi^{-4 i}\right)=\sum_{j \geqslant 1} a_{i, j}^{\prime} T^{-i-j}=\sum_{j \geqslant 1} m_{4 i, j} T^{-j} .
$$

Therefore $a_{i, j}^{\prime}=m_{4 i, i+j}=a_{i, j}$, and

$$
H\left(\xi^{-4 i}\right)=\sum_{j \geq 1} a_{i, j} T^{-i-j}
$$

We can argue similarly to show that $H\left(\xi^{-(4 i+1)}\right)=\Sigma_{j \geq 1} b_{i, j} T^{-i-j}$.

In order to derive the modular equation we first need some preliminary results. Following Kolberg [5] we define

$$
\alpha=-q^{-2} Q_{0}, \quad \beta=q^{-1} Q_{1} \quad \text { and } \quad \gamma=-q^{3} Q_{5}
$$

From Lemma (2.1) we have

(3.6) $\xi(q)=\frac{E(q)}{q^{2} E\left(q^{49}\right)}=q^{-2} Q_{0}-q^{-1} Q_{1}-1+q^{3} Q_{5}=-(\alpha+\beta+\gamma+1)$

https://doi.org/10.1017/S1446788700025386 Published online by Cambridge University Press 
From (2.2) and (2.3) we obtain

$$
\left\{\begin{aligned}
\alpha \beta^{2}+\alpha^{2}+\gamma & =0 \\
\beta \gamma^{2}+\beta^{2}+\alpha & =0 \\
\gamma \alpha^{2}+\gamma^{2}+\beta & =0 \\
\alpha \beta \gamma & =1
\end{aligned}\right.
$$

Let

$$
y_{1}=\alpha^{3} \beta, \quad y_{2}=\beta^{3} \gamma \text { and } y_{3}=\gamma^{3} \alpha .
$$

Then, by (3.7) we easily find

$$
\begin{gathered}
y_{1} y_{2}=-y_{1}-1, \quad y_{2} y_{3}=-y_{2}-1, \quad y_{3} y_{1}=-y_{3}-1, \quad y_{1} y_{2} y_{3}=1, \\
\alpha^{2} \beta^{3}=-y_{1}-1, \quad \beta^{2} \gamma^{3}=-y_{2}-1, \quad \gamma^{2} \alpha^{3}=-y_{3}-1, \\
\alpha \beta^{5}=y_{1}-y_{2}+1, \quad \beta \gamma^{5}=y_{2}-y_{3}+1, \quad \gamma \boldsymbol{\alpha}^{5}=y_{3}-y_{1}+1, \\
\alpha^{7}=-y_{1}^{2}+y_{1}-y_{3}-1, \quad \beta^{7}=-y_{2}^{2}+y^{2}-y_{1}-1, \\
\gamma^{7}=-y_{3}^{2}+y^{3}-y_{2}-1 .
\end{gathered}
$$

The following lemma is $5.20,5.21$ and 5.14 of Kolberg's paper.

LeMma (3.9).

$$
\begin{aligned}
y_{1}+y_{2}+y_{3} & =-T-8, \\
y_{1} y_{2}+y_{2} y_{3}+y_{3} y_{1} & =T+5, \\
y_{1} y_{2} y_{3} & =1,
\end{aligned}
$$

where $T$ is defined in (3.3) and the $y_{i}$ are defined in (3.5) and (3.8).

Proof. Let $\omega^{7}=1, \omega \neq 1$. Then, from Lemma (2.5),

$$
\begin{aligned}
T^{2} & =\prod_{i=0}^{6} \xi\left(\omega^{i} q\right)=-\prod_{i=0}^{6}\left[1+\omega^{3 i} \gamma+\omega^{5 i} \alpha+\omega^{6 i} \beta\right] \\
& =-\operatorname{det}\left[\begin{array}{ccccccc}
1 & 0 & 0 & \gamma & 0 & \alpha & \beta \\
\beta & 1 & 0 & 0 & \gamma & 0 & \alpha \\
\alpha & \beta & 1 & 0 & 0 & \gamma & 0 \\
0 & \alpha & \beta & 1 & 0 & 0 & \gamma \\
\gamma & 0 & \alpha & \beta & 1 & 0 & 0 \\
0 & \gamma & 0 & \alpha & \beta & 1 & 0 \\
0 & 0 & \gamma & 0 & \alpha & \beta & 1
\end{array}\right] \\
& =\left(y_{1}+y_{2}+y_{3}+8\right)^{2},
\end{aligned}
$$


using (3.8) in the evaluation of the determinant. So,

$$
y_{1}+y_{2}+y_{3}+8= \pm T \text {. }
$$

We now calculate the first term in the expansion of each $y_{i} . y_{1}=\alpha^{3} \beta=-q^{-7} Q_{3}^{3} Q_{1}$ $=-q^{-7}+\cdots, y_{2}=\beta^{2} \gamma=-Q_{1}^{3} Q_{5}=-1+\cdots, y_{3}=\gamma^{3} \alpha=q^{7} Q_{5}^{3} Q_{0}=q^{7}+\cdots$.

We therefore have to take the - sign in (3.11) so,

$$
\begin{gathered}
y_{1}+y_{2}+y_{3}=-T-8, \\
y_{1} y_{2}+y_{2} y_{3}+y_{3} y_{1}=-y_{1}-y_{2}-y_{3}-3=T+5, \\
y_{1} y_{2} y_{3}=(\alpha \beta \gamma)^{4}=1
\end{gathered}
$$

and the lemma is proved.

LEMMA (3.12).

$$
\begin{array}{ll}
H(\xi)=-1, & H\left(\xi^{4}\right)=-4 T-7, \\
H\left(\xi^{2}\right)=1, & H\left(\xi^{5}\right)=10 T+49, \\
H\left(\xi^{3}\right)=-7, & H\left(\xi^{6}\right)=49 .
\end{array}
$$

Proof. From (3.5) and (3.7), $H(\alpha+\beta+\gamma)=0, H\left((\alpha+\beta+\gamma)^{2}\right)=0$, $H\left((\alpha+\beta+\gamma)^{3}\right)=6 \alpha \beta \gamma=6$ and using (3.5), (3.7) and Lemma (3.9),

$$
H\left((\alpha+\beta+\gamma)^{4}\right)=4\left(\alpha^{3} \beta+\beta^{3} \gamma+\gamma^{3} \alpha\right)=4\left(y_{1}+y_{2}+y_{3}\right)=-4 T-32 .
$$

Similarly, $H\left((\alpha+\beta+\gamma)^{5}\right)=10 T+50, H\left((\alpha+\beta+\gamma)^{6}\right)=108$, so $H\left(\xi^{1}\right)=$ $H(-(\alpha+\beta+\gamma)-1)=-1$ and the results for the other $H\left(\xi^{j}\right)$ follow in the same way.

Lemma (3.13). $H_{3}\left(\xi^{3}\right)=0, H_{5}\left(\xi^{3}\right)=0, H_{6}\left(\xi^{3}\right)=0$.

Proof. From (3.7) we have $H_{3}(\alpha+\beta+\gamma)=\gamma, H_{3}\left((\alpha+\beta+\gamma)^{2}\right)=\alpha^{2}$, $H_{3}\left((\alpha+\beta+\gamma)^{3}\right)=3 \alpha \beta^{2}$, so

$$
\begin{aligned}
H_{3}\left(\xi^{3}\right) & =H_{3}\left(-(\alpha+\beta+\gamma)^{3}-3(\alpha+\beta+\gamma)^{2}-3(\alpha+\beta+\gamma)-1\right) \\
& =-3 \alpha \beta^{2}-3 \alpha^{2}-3 \gamma=0 .
\end{aligned}
$$

Similarly, $H_{5}\left(\xi^{3}\right)=-3 \beta \gamma^{2}-3 \beta^{2}-3 \alpha=0, H_{6}\left(\xi^{3}\right)=-3 \gamma \alpha^{2}-3 \gamma^{2}-3 \beta=0$.

Lemma (3.14) (the Modular Equation of Seventh Order).

$$
\begin{aligned}
T^{2}= & \left(7 \xi^{3}+35 \xi^{2}+49 \xi\right) T+\xi^{7}+7 \xi^{6}+21 \xi^{5} \\
& +49 \xi^{4}+147 \xi^{3}+343 \xi^{2}+343 \xi
\end{aligned}
$$

where $T$ and $\xi$ are defined in (3.3). 
Proof. For $0 \leqslant i \leqslant 6$ define $\xi_{i}(q)=\xi\left(\omega^{i} q\right)$ where $\omega^{7}=1, \omega \neq 1$. Then

$$
H\left(\xi^{j}\right)=\frac{1}{7} \sum_{i=0}^{6} \xi^{j}\left(\omega^{i} q\right)=\frac{1}{7} \sum_{i=0}^{6} \xi_{i}^{j}
$$

Let $P_{j}=\xi_{0}^{j}+\cdots+\xi_{6}^{j}$. From Lemma (3.12), $P_{1}=-7, P_{2}=7, P_{3}=-49, P_{4}=$ $-28 T-49, P_{5}=70 T+343, P_{6}=343$. Let $S_{j}$ be the symmetric functions of degree $j$ in the $\xi_{i}$. Then, from standard formulae it follows that $S_{1}=-7, S_{2}=21$, $S_{3}=-49, S_{4}=7 T+147, S_{5}=-35 T-343, S_{6}=49 T+343$. Finally, from (3.10), $S_{7}=T^{2}$. Hence the $\xi_{i}$ are the roots of

$$
\begin{aligned}
& X^{7}+7 X^{6}+21 X^{5}+49 X^{4}+(7 T+147) X^{3} \\
& \quad+(35 T+343) X^{2}+(49 T+343) X-T^{2}=0
\end{aligned}
$$

But $\xi_{0}=\xi$ and the lemma is proved.

We are now in a position to prove Lemma (3.1). From Lemma (3.14) it follows that

$$
\begin{aligned}
\xi^{-i}= & {\left[7 \xi^{-(i-3)}+35 \xi^{-(i-2)}+49 \xi^{-(i-1)}\right] T^{-1} } \\
& +\left[\xi^{-(i-7)}+7 \xi^{-(i-6)}+21 \xi^{-(i-5)}\right. \\
& \left.\quad+49 \xi^{-(i-4)}+147 \xi^{-(i-3)}+343 \xi^{-(i-2)}+343 \xi^{-(i-1)}\right] T^{-2}
\end{aligned}
$$

Picking out those terms in which the power of $q$ is congruent to $0 \bmod 7$ we obtain:

$$
\begin{aligned}
H\left(\xi^{-i}\right)= & {\left[7 H\left(\xi^{-(i-3)}\right)+35 H\left(\xi^{-(i-2)}\right)+49 H\left(\xi^{-(i-1)}\right)\right] T^{-1} } \\
& +\left[H\left(\xi^{-(i-7)}\right)+7 H\left(\xi^{-(i-6)}\right)+21 H\left(\xi^{-(i-5)}\right)+49 H\left(\xi^{-(i-4)}\right)\right. \\
& \left.+147 H\left(\xi^{-(i-3)}\right)+343 H\left(\xi^{-(i-2)}\right)+343 H\left(\xi^{-(i-1)}\right)\right] T^{-2}
\end{aligned}
$$

From (3.15) and Lemma (3.12) we have

$$
\begin{aligned}
H\left(\xi^{-1}\right)=(7-35+49) T^{-1}+[49+7(10 T+49)+21(-4 T-7) \\
+49(-7)+147-343+343] T^{-2}
\end{aligned}
$$

or

$$
H\left(\xi^{-1}\right)=7 T^{-1}+49 T^{-2}
$$


So (3.2) is true for $i=1$. Similarly it is easily verified that (3.2) holds for $i=2,3,4,5,6,7$ from (3.15) together with Lemma (3.12). Further it is clear from (3.15) that we can write

$$
H\left(\xi^{-i}\right)=\sum_{j \geqslant 1} m_{i, j}^{\prime} T^{-j}
$$

Already we have $m_{i, j}^{\prime}=m_{i, j}$ for $1 \leqslant i \leqslant 7$. It follows from (3.15) that $m_{i, 1}^{\prime}=0$ for $i \geqslant 4$ and $m_{i, 2}^{\prime}=0$ for $i \geqslant 8$. From (3.15), we have for $i \geqslant 8$

$$
\begin{aligned}
& \sum_{j \geqslant 3} m_{i, j}^{\prime} T^{-j}= \sum_{j \geqslant 1}\left(7 m_{i-3, j}^{\prime}+35 m_{i-2, j}^{\prime}+49 m_{i-1, j}^{\prime}\right) T^{-j-1} \\
&+\sum_{j \geqslant 1}\left(m_{i-7, j}^{\prime}+7 m_{i-6, j}^{\prime}+21 m_{i-5, j}^{\prime}+49 m_{i-4, j}^{\prime}\right. \\
&\left.\quad+147 m_{i-3, j}^{\prime}+343 m_{i-2, j}^{\prime}+343 m_{i-1, j}^{\prime}\right) T^{-j-2} \\
&=\sum_{j \geqslant 3}\left(7 m_{i-3, j-1}^{\prime}+35 m_{i-2, j-1}^{\prime}+49 m_{i-1, j-1}^{\prime}\right. \\
&\left.\quad+m_{i-1, j-2}^{\prime}+\cdots+343 m_{i-1, j-2}^{\prime}\right) T^{-j} .
\end{aligned}
$$

Hence, for $i \geqslant 8, j \geqslant 3$

$$
\begin{aligned}
m_{i, j}^{\prime}= & 7 m_{i-3, j-1}^{\prime}+35 m_{i-2, j-1}^{\prime}+49 m_{i-1, j-1}^{\prime}+m_{i-7, j-2}^{\prime} \\
& +7 m_{i-6, j-2}^{\prime}+21 m_{i-5, j-2}^{\prime}+49 m_{i-4, j-2}^{\prime}+147 m_{i-3, j-2}^{\prime} \\
& +343 m_{i-2, j-2}^{\prime}+343 m_{i-1, j-2}^{\prime} .
\end{aligned}
$$

Therefore $m_{i, j}^{\prime}=m_{i, j}$ for every $i, j \geqslant 1$.

Before proving our main theorem we need one more lemma.

LEMMA (3.17). $\lambda_{\alpha}$, the reciprocal modulo $7^{\alpha}$ of 24 , satisfies $\lambda_{1}=5$ and for $\alpha \geqslant 1$,

$$
\lambda_{\alpha+1}= \begin{cases}6 \times 7^{\alpha}+\lambda_{\alpha}, & \alpha \text { odd }, \\ 4 \times 7^{\alpha}+\lambda_{\alpha}, & \alpha \text { even } .\end{cases}
$$

Proof.

$$
\lambda_{\alpha}= \begin{cases}\frac{1}{24}\left(17 \times 7^{\alpha}+1\right), & \alpha \text { odd } \\ \frac{1}{24}\left(23 \times 7^{\alpha}+1\right), & \alpha \text { even }\end{cases}
$$

since this is an integer which satisfies $0<\lambda_{\alpha}<7^{\alpha}$ and $24 \lambda_{\alpha} \equiv 1 \bmod 7^{\alpha}$. It is easily shown that this $\lambda_{\alpha}$ satisfies the recurrence. 
We are now in a position to prove Theorem (1.6). For convenience we write the theorem in the following equivalent form

$$
\sum_{n \geqslant 0} p\left(7^{\alpha} n+\lambda_{\alpha}\right) q^{n}= \begin{cases}{\left[\sum_{i \geqslant 1} x_{\alpha, i} T^{i} \xi^{-4 i}\right] / q E\left(q^{7}\right),} & \alpha \text { odd } \\ {\left[\sum_{i \geqslant 1} x_{\alpha, i} T^{i} \xi^{-4 i-1}\right] / q^{3} E\left(q^{49}\right),} & \alpha \text { even }\end{cases}
$$

where $T$ and $\xi$ are defined in (3.3).

We have

$$
\sum_{n \geqslant 0} p(n) q^{n}=\frac{1}{E(q)}=\frac{\xi^{-1}}{q^{2} E\left(q^{49}\right)} .
$$

Picking out those terms in which the power of $q$ is congruent to $5 \bmod 7$, we have by (3.16)

$$
\sum_{n \geqslant 0} p(7 n+5) q^{7 n+5}=\frac{H\left(\xi^{-1}\right)}{q^{2} E\left(q^{49}\right)}=\frac{7 T^{-1}+49 T^{-2}}{q^{2} E\left(q^{49}\right)}
$$

or

$$
\sum_{n \geqslant 0} p(7 n+5) q^{7 n}=\frac{7 T^{-1}+49 T^{-2}}{q^{7} E\left(q^{49}\right)}
$$

Now

$$
T\left(q^{1 / 7}\right)=\frac{E(q)^{4}}{q E\left(q^{7}\right)^{4}}=\frac{q^{8} \xi^{4} E\left(q^{49}\right)^{4}}{q E\left(q^{7}\right)^{4}}=\xi^{4} T^{-1},
$$

so

$$
T^{-1}\left(q^{1 / 7}\right)=T \xi^{-4}(q)
$$

So we have

$$
\sum_{n \geqslant 0} p(7 n+5) q^{n}=\frac{7 T \xi^{-4}+49 T^{2} \xi^{-8}}{q E\left(q^{7}\right)} .
$$

Substituting $T=q^{-7} E\left(q^{7}\right)^{4} / E\left(q^{49}\right)^{4}$ and $\xi^{-1}=q^{2} E\left(q^{49}\right) / E(q)$ we obtain Ramanujan's result:

$$
\sum_{n \geqslant 0} p(7 n+5) q^{n}=7 \frac{E\left(q^{7}\right)^{3}}{E(q)^{4}}+49 q \frac{E\left(q^{7}\right)^{7}}{E(q)^{8}},
$$

which is the case $\alpha=1$ of Theorem (1.6). 
We now proceed by induction on $\alpha$. Suppose $\alpha$ is odd and

$$
\sum_{n \geqslant 0} p\left(7^{\alpha} n+\lambda_{\alpha}\right) q^{n}=\left[\sum_{i \geqslant 1} x_{\alpha, i} T^{i} \xi^{-4 i}\right] / q E\left(q^{7}\right) .
$$

Picking out those terms in which the power of $q$ is congruent to $6 \bmod 7$ we have by Lemma (3.4),

$$
\begin{aligned}
\sum_{n \geqslant 0} p\left(7^{\alpha}(7 n+6)+\lambda_{\alpha}\right) q^{7 n+6} & =\left[\sum_{i \geqslant 1} x_{\alpha, i} T^{i} H\left(\xi^{-4 i}\right)\right] / q E\left(q^{7}\right) \\
& =\left[\sum_{i \geqslant 1} x_{\alpha, i} T^{i} \sum_{j \geqslant 1} a_{i, j} T^{-i-j}\right] / q E\left(q^{7}\right) \\
& =\left[\sum_{j \geqslant 1}\left(\sum_{i \geqslant 1} x_{\alpha, i} a_{i, j}\right) T^{-j}\right] / q E\left(q^{7}\right) .
\end{aligned}
$$

It follows from (1.7) and Lemma (3.17) that

$$
\sum_{n \geqslant 0} p\left(7^{\alpha+1} n+\lambda_{\alpha+1}\right) q^{7 n}=\left[\sum_{i \geqslant 1} x_{\alpha+1, i} T^{-i}\right] / q^{7} E\left(q^{7}\right)
$$

From (3.3) and (4.2) we have

$$
\begin{aligned}
\sum_{n \geqslant 0} p\left(7^{\alpha+1} n+\lambda_{\alpha+1}\right) q^{n} & =\left[\sum_{i \geqslant 1} x_{\alpha+1, i} T^{i} \xi^{-4 i}\right] / q E(q) \\
& =\left[\sum_{i \geqslant 1} x_{\alpha+1, i} T^{i} \xi^{-4 i-1}\right] / q^{3} E\left(q^{49}\right) .
\end{aligned}
$$

Now suppose $\alpha$ is even and

$$
\sum_{n \geqslant 0} p\left(7^{\alpha} n+\lambda_{\alpha}\right) q^{n}=\left[\sum_{i \geqslant 1} x_{\alpha, l} T^{i} \xi^{-4 i-1}\right] / q^{3} E\left(q^{49}\right) .
$$

Picking out those terms in which the power of $q$ is congruent to $4 \bmod 7$, we have by Lemma (3.4),

$$
\begin{aligned}
\sum_{n \geqslant 0} p\left(7^{\alpha}(7 n+4)+\lambda_{\alpha}\right) q^{7 n+4} & =\left[\sum_{i \geqslant 1} x_{\alpha, i} T^{i} H\left(\xi^{-4 i-1}\right)\right] / q^{3} E\left(q^{49}\right) \\
& =\left[\sum_{i \geqslant 1} x_{\alpha, i} T^{i} \sum_{j \geqslant 1} b_{i, j} T^{-i-j}\right] / q^{3} E\left(q^{49}\right) \\
& =\left[\sum_{j \geqslant 1}\left(\sum_{i \geqslant 1} x_{\alpha, i} b_{i, j}\right) T^{-j}\right] / q^{3} E\left(q^{49}\right) .
\end{aligned}
$$

It follows from (1.7) and Lemma (3.17) that

$$
\sum_{n \geqslant 0} p\left(7^{\alpha+1} n+\lambda_{\alpha+1}\right) q^{7 n}=\left[\sum_{i \geqslant 1} x_{\alpha+1, i} T^{-i}\right] / q^{7} E\left(q^{49}\right) .
$$


From (4.2) we have

$$
\sum_{n \geqslant 0} p\left(7^{\alpha+1} n+\lambda_{\alpha+1}\right) q^{n}=\left[\sum_{i \geqslant 1} x_{\alpha+1, i} T^{i} \xi^{-4 i}\right] / q E\left(q^{7}\right)
$$

This completes the proof of Theorem (1.6).

\section{5}

We now turn to Watson's results. Let $\nu(n)$ denote the exact power of 7 dividing $n$. Then

LemMA (5.1). $\nu\left(m_{i, j}\right) \geqslant\left[\frac{1}{4}(7 j-2 i-1)\right]$.

Proof. Consider the matrix $V=\left(\nu\left(m_{i, j}\right)\right)_{i, j \geqslant 1}$. The first seven rows of $V$ are:

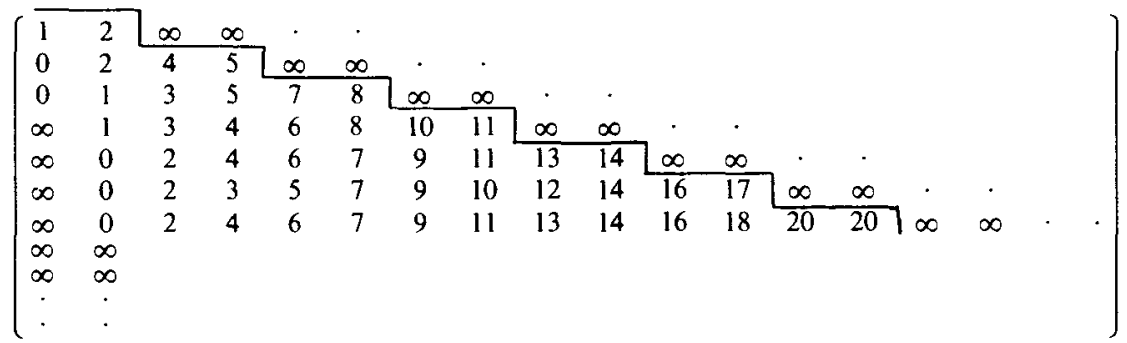

Define the matrix $N=\left(\nu_{i, j}\right)_{i, j \geqslant 1}$ by $\nu_{i, j}=[(7 j-2 i-1) / 4]$. The first seven rows of $N$ are:

$$
\left(\begin{array}{cccccccccccccccc}
\hline 1 & 2 & 4 & 6 & 8 & 9 & 11 & 13 & 15 & 16 & 18 & 20 & 22 & 23 & . & . \\
0 & 2 & 4 & 5 & 7 & 9 & 11 & 12 & 14 & 16 & 18 & 19 & 21 & 23 & . & . \\
0 & 1 & 3 & 5 & 7 & 8 & 10 & 12 & 14 & 15 & 17 & 19 & 21 & 22 & . & . \\
-1 & 1 & 3 & 4 & 6 & 8 & 10 & 11 & 13 & 15 & 17 & 18 & 20 & 22 & . & . \\
-1 & 0 & 2 & 4 & 6 & 7 & 9 & 11 & 13 & 14 & 16 & 18 & 20 & 21 & . & . \\
-2 & 0 & 2 & 3 & 5 & 7 & 9 & 10 & 12 & 14 & 16 & 17 & 19 & 21 & . & . \\
-2 & -1 & 1 & 3 & 5 & 6 & 8 & 10 & 12 & 13 & 15 & 17 & 19 & 20 & . & . \\
. & . & . & . & . & . & . & . & . & . & . & . & . & . &
\end{array}\right)
$$

Observe that for $i \leqslant 7$ and for $i>7, j \leqslant 2, \nu\left(m_{i, j}\right) \geqslant \nu_{i, j}$. From (1.10) it follows that for $i>7, j>2$,

$$
\begin{array}{r}
\nu\left(m_{i, j}\right) \geqslant \min \left\{\nu\left(m_{i-3, j-1}\right)+1, \nu\left(m_{i-2, j-1}\right)+1, \nu\left(m_{i-1, j-1}\right)+2,\right. \\
\nu\left(m_{i-7, j-2}\right), \nu\left(m_{i-6, j-2}\right)+1, \nu\left(m_{i-5, j-2}\right)+1, \\
\nu\left(m_{i-4, j-2}\right)+2, \nu\left(m_{i-3, j-2}\right)+2, \\
\left.\nu\left(m_{i-2, j-2}\right)+3, \nu\left(m_{i-1, j-2}\right)+3\right\}
\end{array}
$$


while, as is easily checked

$$
\begin{aligned}
\nu_{i, j}=\min \left\{\nu_{i-3, j-1}+1, \nu_{i-2, j-1}+1, \nu_{i-1, j-1}+2,\right. \\
\\
\boldsymbol{v}_{i-7, j-2}, \nu_{i-6, j-2}+1, \nu_{i-5, j-2}+1, \\
\left.\boldsymbol{\nu}_{i-4, j-2}+2, \nu_{i-3, j-2}+2, \nu_{i-2, j-2}+3, \nu_{i-1, j-2}+3\right\} .
\end{aligned}
$$

Lemma (5.1) follows by induction.

Lemma (5.2).

$$
\nu\left(a_{i, j}\right) \geqslant\left[\frac{7 j-i-1}{4}\right], \quad \nu\left(b_{i, j}\right) \geqslant\left[\frac{7 j-i-3}{4}\right] .
$$

Proof. From (5.1) we have

$$
\begin{gathered}
\nu\left(a_{i, j}\right)=\nu\left(m_{4 i, i+j}\right) \geqslant\left[\frac{7(i+j)-8 i-1}{4}\right]=\left[\frac{7 j-i-1}{4}\right], \\
\nu\left(b_{i, j}\right)=\nu\left(m_{4 i+1, i+j}\right) \geqslant\left[\frac{7(i+j)-2(4 i+1)-1}{4}\right]=\left[\frac{7 j-i-3}{4}\right] .
\end{gathered}
$$

$\operatorname{LemMA~(5.3).~} \nu\left(x_{1,1}\right)=1, \nu\left(x_{1,2}\right)=2$ and for $\beta \geqslant 1$,

$$
\begin{gathered}
\nu\left(x_{2 \beta, j}\right) \geqslant(\beta+1)+\left[\frac{7 j-6}{4}\right], \\
\nu\left(x_{2 \beta+1, j}\right) \geqslant(\beta+1)+\left[\frac{7 j-4}{4}\right] .
\end{gathered}
$$

Proof. $x_{1}=(7,49,0,0, \ldots)$, so $\nu\left(x_{1,1}\right)=1, \quad v\left(x_{1,2}\right)=2$. We have $x_{2, j}=$ $\sum_{i \geq 1} x_{1, i} a_{i, j}=7 a_{1, j}+49 a_{2, j}$ so

$$
\begin{aligned}
\nu\left(x_{2, j}\right) & \geqslant \min \left\{1+\nu\left(a_{1, j}\right), 2+\nu\left(a_{2, j}\right)\right\} \\
& \geqslant \min \left\{1+\left[\frac{7 j-2}{4}\right], 2+\left[\frac{7 j-3}{4}\right]\right\} \\
& =2+\left[\frac{7 j-6}{4}\right], \quad \text { as required. }
\end{aligned}
$$

Now suppose $\beta \geqslant 1$ and $\nu\left(x_{2 \beta, i}\right) \geqslant(\beta+1)+[(7 i-6) / 4]$. We have $x_{2 \beta+1, j}=$ $\sum_{i \geqslant 1} x_{2 \beta, i} b_{i, j}$ so

$$
\begin{aligned}
\nu\left(x_{2 \beta+1, j}\right) & \geqslant \min _{i \geqslant 1}\left\{\nu\left(x_{2 \beta, i}\right)+\nu\left(b_{i, j}\right)\right\} \\
& \geqslant \min _{i \geqslant 1}\left\{(\beta+1)+\left[\frac{7 i-6}{4}\right]+\left[\frac{7 j-i-3}{4}\right]\right\} \\
& =(\beta+1)+\left[\frac{7 j-4}{4}\right], \quad \text { as required. }
\end{aligned}
$$


Finally, given $\nu\left(x_{2 \beta+1, i}\right) \geqslant(\beta+1)+[(7 j-4) / 4]$, we can argue similarly to show that

$$
\nu\left(x_{2 \beta+2, j}\right) \geqslant(\beta+2)+\left[\frac{7 j-6}{4}\right] .
$$

Lemma (5.3) follows by induction. It follows that $x_{2 \beta-1, i} \equiv 0 \bmod 7^{\beta}$ and $x_{2 \beta, i} \equiv 0 \bmod 7^{\beta+1}$, which together with Theorem (1.6) yield the following theorem.

THEOREM (5.4). For $\beta \geqslant 1$,

$$
\begin{gathered}
p\left(7^{2 \beta-1} n+\lambda_{2 \beta-1}\right) \equiv 0 \bmod 7^{\beta}, \\
p\left(7^{2 \beta} n+\lambda_{2 \beta}\right) \equiv 0 \bmod 7^{\beta+1}
\end{gathered}
$$

TheOrem (5.5). For $\beta \geqslant 1$,

$$
\begin{aligned}
p\left(7^{2 \beta} n+\lambda_{2 \beta}-4.7^{2 \beta-1}\right) & \equiv p\left(7^{2 \beta-1} n+\lambda_{2 \beta}-2.7^{2 \beta-1}\right) \\
& \equiv p\left(7^{2 \beta} n+\lambda_{2 \beta}-7^{2 \beta-1}\right) \equiv 0 \bmod 7^{\beta+1} .
\end{aligned}
$$

Proof. From (4.1) we have

$$
\sum_{n \geqslant 0} p\left(7^{2 \beta-1} n+\lambda_{2 \beta-1}\right) q^{n}=\left[\sum_{i \geqslant 1} x_{2 \beta-1, i} T^{i} \xi^{-4 i}\right] / q E\left(q^{7}\right)
$$

If we pick out those terms in which the power of $q$ is congruent to $k \bmod 7$ we have

$$
\begin{aligned}
\sum_{n \geq 0} p\left(7^{2 \beta-1}(7 n+k)+\lambda_{2 \beta-1}\right) q^{7 n+k} \\
=\left[\sum_{i \geqslant 1} x_{2 \beta-1, i} T^{i} H_{k+1}\left(\xi^{-4 i}\right)\right] / q E\left(q^{7}\right) .
\end{aligned}
$$

From Lemma (3.17) it follows that

$$
\sum_{n \geqslant 0} p\left(7^{2 \beta} n+\lambda_{2 \beta}+(k-6) 7^{2 \beta-1}\right) q^{7 n+k}=\left[\sum_{i \geqslant 1} x_{2 \beta-1, i} T^{i} H_{k+1}\left(\xi^{-4 i}\right)\right] / q E\left(q^{7}\right) .
$$

From Lemma (5.3) $\nu\left(x_{1,1}\right)=1, \nu\left(x_{1,2}\right)=2$ and for $\beta \geqslant 2$,

$$
\nu\left(x_{2 \beta-1, i}\right) \geqslant \beta+\left[\frac{7 i-4}{4}\right] \text {. }
$$


So modulo $7^{\beta+1}$ we have

$$
\begin{gathered}
\sum_{n \geq 0} p\left(7^{2 \beta} n+\lambda_{2 \beta}+(k-6) 7^{2 \beta-1}\right) q^{7 n+k} \\
\equiv\left[x_{2 \beta-1,1} T H_{k+1}\left(\xi^{-4}\right)\right] / q E\left(q^{7}\right) .
\end{gathered}
$$

From the modular equation (3.14) we have

$$
\begin{aligned}
\xi^{-4}=\xi^{3} T^{-2}+7\left(\xi^{-1} T^{-1}+5 \xi^{-2} T^{-1}\right. & +7 \xi^{-3} T^{-1}+\xi^{2} T^{-2}+3 \xi T^{-2} \\
& \left.+7 T^{-2}+21 \xi^{-1} T^{-2}+49 \xi^{-2} T^{-2}+49 \xi^{-3} T^{-2}\right) .
\end{aligned}
$$

Picking out those terms in which the power of $q$ is congruent to $(k+1) \bmod 7$ we have

$$
H_{k+1}\left(\xi^{-4}\right) \equiv T^{-2} H_{k+1}\left(\xi^{3}\right) \bmod 7 .
$$

But form (3.13) we have $H_{k+1}\left(\xi^{3}\right)=0$ for $k=2,4$ and 5, so

$$
H_{k+1}\left(\xi^{-4}\right) \equiv 0 \bmod 7 \quad \text { for } k=2,4 \text { and } 5 .
$$

Since $x_{2 \beta-1,1} \equiv 0 \bmod 7^{\beta}$ we obtain

$$
\sum_{n \geqslant 0} p\left(7^{2 \beta} n+\lambda_{2 \beta}+(k-6) 7^{2 \beta-1}\right) q^{7 n+k} \equiv 0 \bmod 7^{\beta+1} \quad \text { for } k=2,4 \text { and } 5,
$$

which is the required result.

\section{6}

We have calculated $\mathbf{x}_{\alpha}$ for $\alpha=1,2$. They are

$$
\begin{aligned}
& \mathbf{x}_{1}=\left(7,7^{2}, 0,0,0, \ldots\right), \\
& \mathbf{x}_{2}=\left(2546 \times 7^{2}, 48934 \times 7^{4}, 1418989 \times 7^{5}, 2488800 \times 7^{7}, 2394438 \times 7^{9},\right. \\
& 1437047 \times 7^{11}, 4043313 \times 7^{12}, 161744 \times 7^{15}, 32136 \times 7^{17}, \\
& \left.\quad 31734 \times 7^{18}, 3120 \times 7^{20}, 204 \times 7^{22}, 8 \times 7^{24}, 7^{25}, 0,0,0, \ldots\right) .
\end{aligned}
$$

Therefore from Theorem (1.6) we have

$$
\sum_{n \geqslant 0} p(7 n+5) q^{n}=7 \frac{E\left(q^{7}\right)^{3}}{E(q)^{4}}+49 q \frac{E\left(q^{7}\right)^{7}}{E(q)^{8}}
$$


which is Ramanujan's result and

$$
\begin{aligned}
\sum_{n \geqslant 0} p(49 n+47) q^{n}= & 2546 \times 7^{2} \frac{E\left(q^{7}\right)^{4}}{E(q)^{5}}+48934 \times 7^{4} q \frac{E\left(q^{7}\right)^{8}}{E(q)^{9}} \\
& +1418989 \times 7^{5} q^{2} \frac{E\left(q^{7}\right)^{12}}{E(q)^{13}}+2488800 \times 7^{7} q^{3} \frac{E\left(q^{7}\right)^{16}}{E(q)^{17}} \\
& +2394438 \times 7^{9} q^{4} \frac{E\left(q^{7}\right)^{20}}{E(q)^{21}}+1437047 \times 7^{11} q^{5} \frac{E\left(q^{7}\right)^{24}}{E(q)^{25}} \\
& +4043313 \times 7^{12} q^{6} \frac{E\left(q^{7}\right)^{28}}{E(q)^{29}}+161744 \times 7^{15} q^{7} \frac{E\left(q^{7}\right)^{32}}{E(q)^{33}} \\
& +32136 \times 7^{17} q^{8} \frac{E\left(q^{7}\right)^{36}}{E(q)^{37}}+31734 \times 7^{18} q^{9} \frac{E\left(q^{7}\right)^{40}}{E(q)^{41}} \\
& +3120 \times 7^{20} q^{10} \frac{E\left(q^{7}\right)^{44}}{E(q)^{45}}+204 \times 7^{22} q^{11} \frac{E\left(q^{7}\right)^{48}}{E(q)^{49}} \\
& +8 \times 7^{24} q^{12} \frac{E\left(q^{7}\right)^{52}}{E(q)^{53}}+7^{25} q^{13} \frac{E\left(q^{7}\right)^{55}}{E(q)^{57}} \cdot
\end{aligned}
$$

This confirms a result of $H$. Zuckerman [8]. We have omitted the calculation of $\mathbf{x}_{\alpha}$ for $\alpha \geqslant 3$, since there are computational difficulties. For an idea of the size of these numbers see Hirschhorn and Hunt [4], who have calculated the first four coefficient vectors corresponding to the generating functions for $p\left(5^{\alpha} n+\delta_{\alpha}\right)$.

I wish to express my thanks to my supervisor, Dr. Michael Hirschhorn, for his help and encouragement in writing this paper.

\section{References}

[1] A. O. L. Atkin, 'Ramanujan congruences for $p_{-k}(n)$ ', Canad.J. Math. 20 (1968), 67-78.

[2] S. Chowla, 'Congruence properties of partitions', J. London Math. Soc. 9 (1934), 247.

[3] H. Gupta, 'A table of partitions', Proc. London Math. Soc. (2) 39 (1935), 142-149.

[4] M. D. Hirschhorn and D. C. Hunt, 'A simple proof of the Ramanujan conjecture for powers of 5', J. Reine Angew. Math. 326 (1981), 1-17.

[5] O. Kolberg, 'Some identities involving the partition function', Math. Scand . 5(1957), 77-92.

[6] S. Ramanujan, 'Some properties of $p(n)$, the number of partitions of $n$ ', Proc. Cambridge Philos. Soc. 19 (1919), 207-210. 
[7] G. N. Watson, 'Ramanujans Vermutung über Zerfällungsanzahlen', J. Reine Angew. Math. 179 (1938), 97-128.

[8] H. S. Zuckerman, 'Identities analogous to Ramanujan's identities involving the partition function', Duke Math. J. 5 (1939), 88-119.

School of Mathematics

University of New South Wales

Post Office Box 1

Kensington, N.S.W. 2033

Australia
The Pennsylvania State University Department of Mathematics University Park, Pennsylvania 16802

U.S.A. 